\title{
Synthesis and Photophysical Properties of
}

\section{Dihydroheptacenes: New Blue Emitting Materials}

\author{
Rajib Mondal, Bipin K. Shah, and Douglas C. Neckers* \\ Center for Photochemical Sciences, \\ Bowling Green State University, \\ Bowling Green, OH 43403
}

neckers@photo.bgsu.edu

\section{Supporting Information}

\section{CONTENTS}

General Experimental Methods.

Figure S1. Fluorescence decay of $\mathbf{1}$.

Figure S2. Fluorescence decay of 2.

Figure S3. Normalized solid state fluorescence spectra recorded from thin films of PMMA containing 2. S4

Figure S4. Normalized solid state fluorescence spectra recorded from thin films of PMMA containing 3. S4

Figure S5. Molecular packing in the crystal structure of $\mathbf{1 .}$

Table S1. Crystal data and structure refinement for 1 . 
Table S2. Atomic coordinates ( $\left.\times 10^{4}\right)$ and equivalent isotropic displacement parameters $\left(\AA^{2} \times 10^{3}\right)$ for 1. S6

Table S3. Bond lengths $[\AA]$ and angles $\left[^{\circ}\right]$ for $\mathbf{1}$.

Table S4. Anisotropic displacement parameters $\left(\AA^{2} \times 10^{3}\right)$ for $\mathbf{1}$.

Table S5. Hydrogen coordinates $\left(\times 10^{4}\right)$ and isotropic displacement parameters $\left(\mathrm{A}^{2} \times 10^{3}\right)$ for $\mathbf{1}$.

Figure S6. ${ }^{1} \mathrm{H}$ NMR spectra of $\mathbf{1}$.

Figure S7. ${ }^{1} \mathrm{H}$ NMR spectra of 2.

Figure S8. ${ }^{1} \mathrm{H}$ NMR spectra of $\mathbf{3}$.

\section{General Experimental Methods}

Solvents and reagents were used as received from commercial suppliers. Reactions that required anhydrous conditions were carried out under argon in oven-dried glassware. Organic solvents were either spectroscopic grade or purified by distillation and dried before use using proper drying reagents.

Mass spectra were recorded on a GCMS instrument equipped with a direct probe. Matrix assisted laser desorption ionization (MALDI) spectra were obtained using a dithranol matrix and the instrument $\left(\mathrm{N}_{2}\right.$ laser, $337 \mathrm{~nm}$ ) operating in the reflector mode. NMR spectra were recorded on a spectrometer with working frequency $300.0 \mathrm{MHz}$ for ${ }^{1} \mathrm{H}$. All measurements were carried out at room temperature unless otherwise specified. 


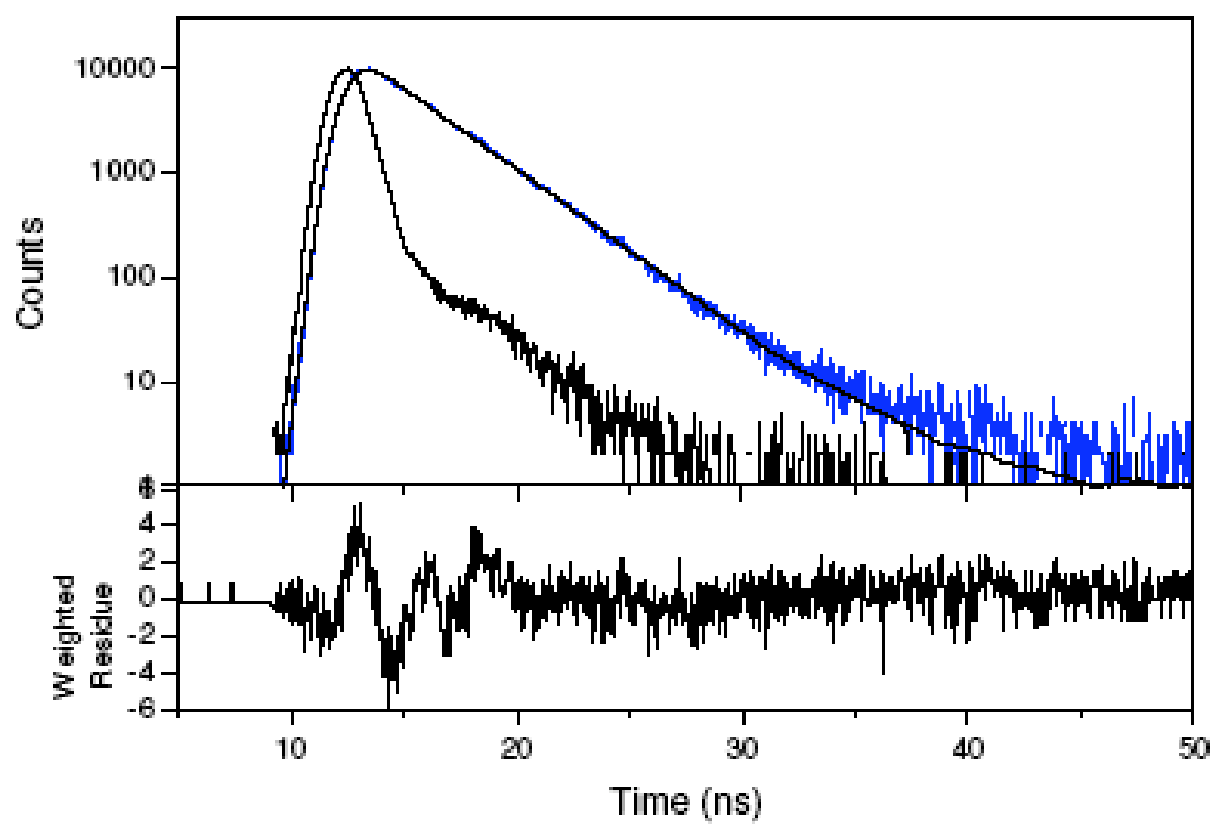

Figure S1. Fluorescence decay of 1 monitored at $\lambda_{\max }=424 \mathrm{~nm}$ in $\mathrm{CH}_{2} \mathrm{Cl}_{2}$; excitation wavelength $=372 \mathrm{~nm}$.

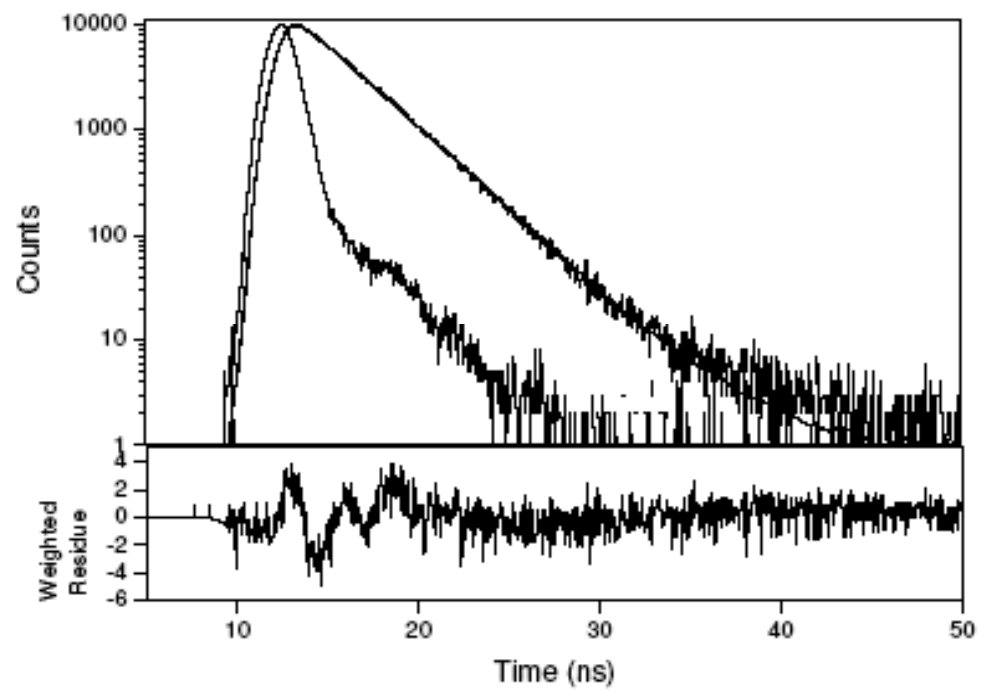

Figure S2. Fluorescence decay of 2 monitored at $\lambda_{\max }=424 \mathrm{~nm}$ in $\mathrm{CH}_{2} \mathrm{Cl}_{2}$; excitation wavelength $=372 \mathrm{~nm}$. 


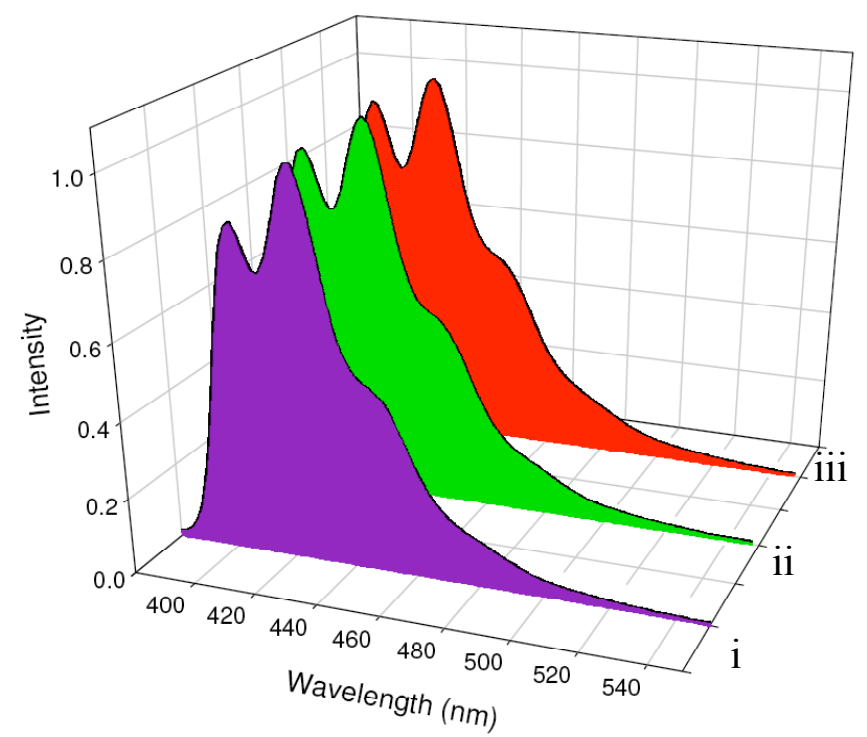

Figure S3. Normalized solid state fluorescence spectra recorded from thin films of PMMA containing 2: (i) pristine, (ii) after exposing the film for 7 days at ambient condition, and (iii) after heating the film at $110{ }^{\circ} \mathrm{C}$ for 24 hours and cooling it down to room temperature.

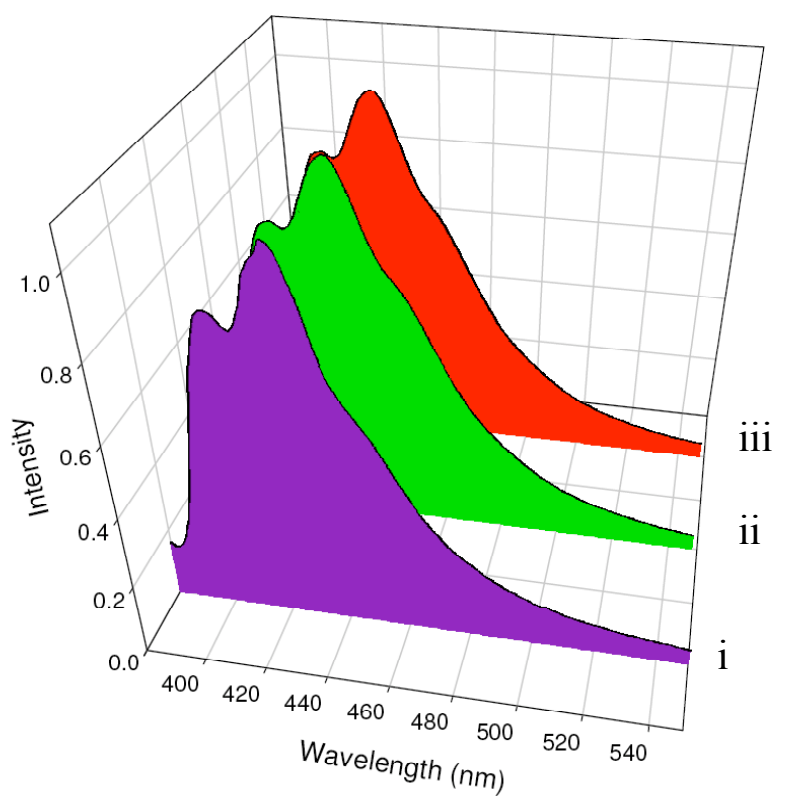

Figure S4. Normalized solid state fluorescence spectra recorded from thin films of PMMA containing 3: (i) pristine, (ii) after exposing the film for 7 days at ambient condition, and (iii) after heating the film at $110{ }^{\circ} \mathrm{C}$ for 24 hours and cooling it down to room temperature. 

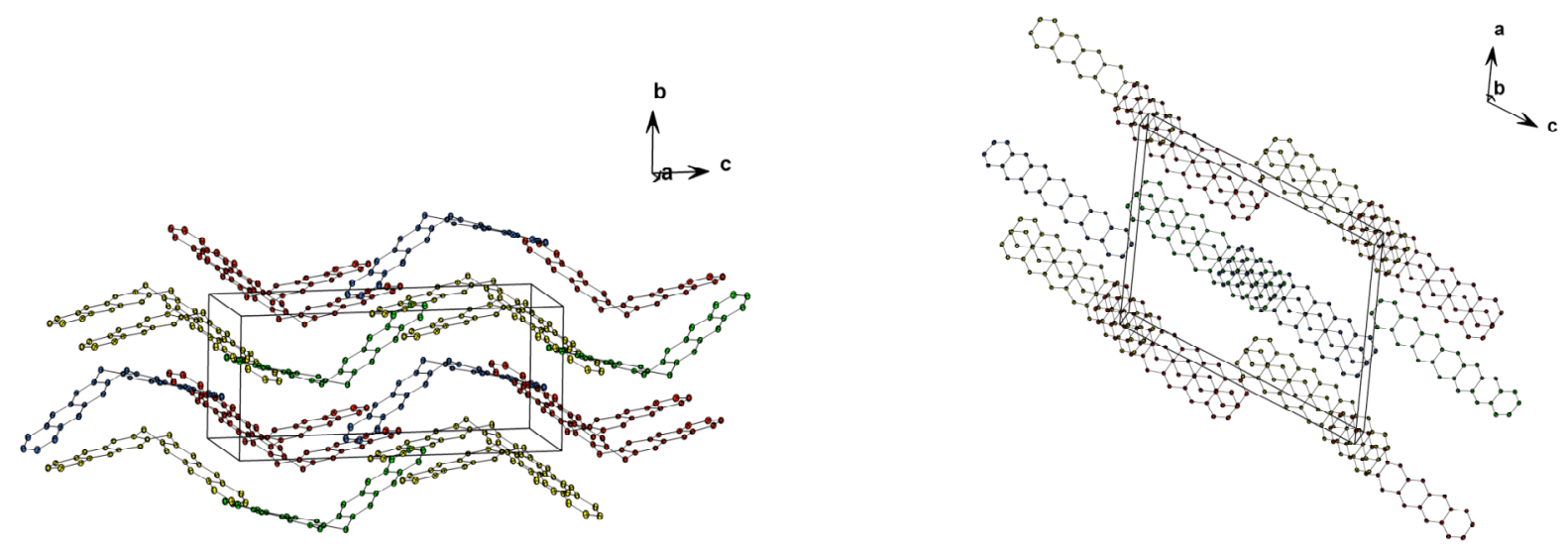

Figure S5. Molecular packing in the crystal structure of $\mathbf{1 .}$

Table S1. Crystal data and structure refinement for $\mathbf{1}$

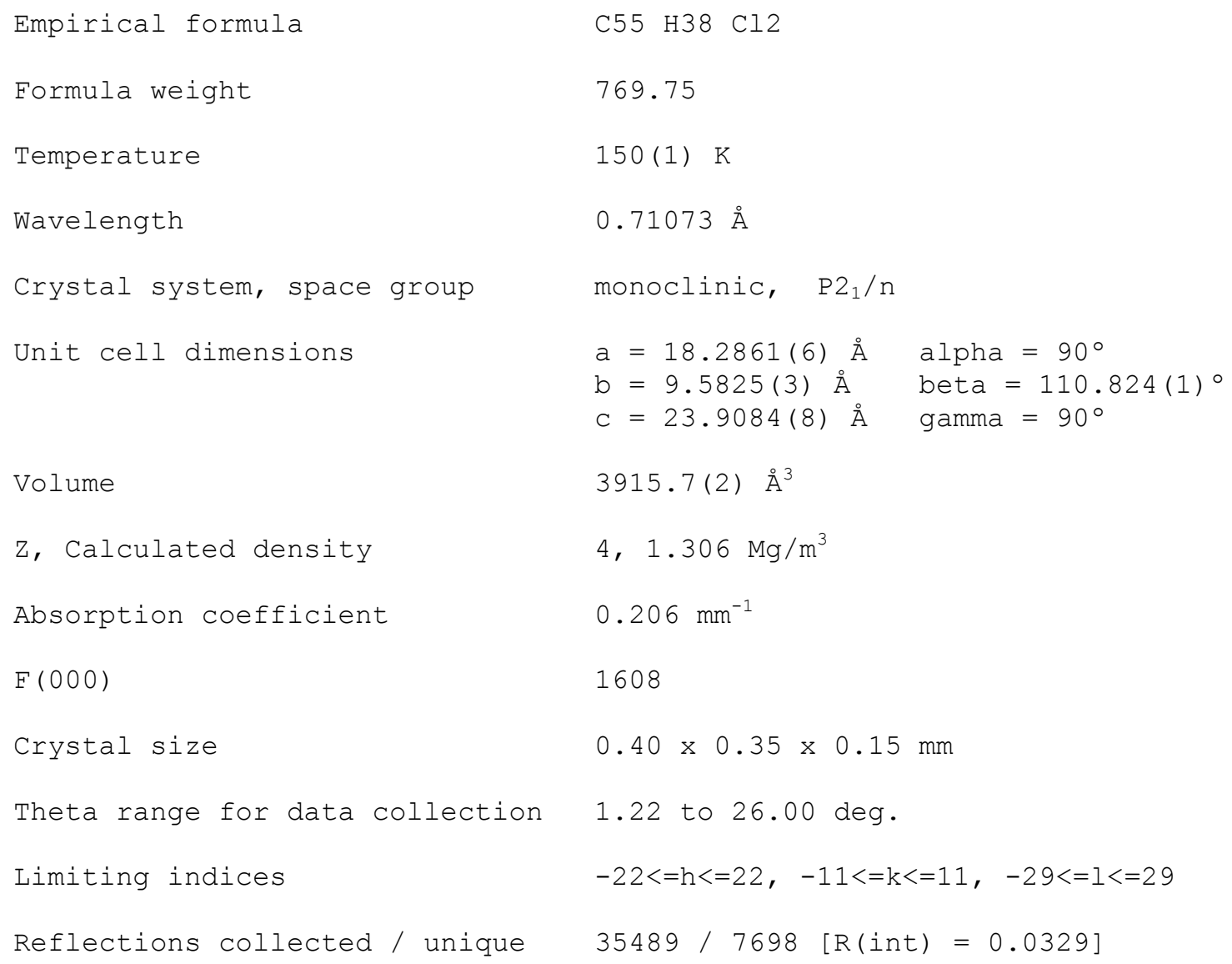




$\begin{array}{ll}\text { Completeness to theta }=26.00 & 100.0 \% \\ \text { Refinement method } & \text { Full-matrix least-squares on } \mathrm{F}^{2} \\ \text { Data / restraints / parameters } & 7698 / 0 / 658 \\ \text { Goodness-of-fit on } \mathrm{F}^{2} & 1.070 \\ \text { Final R indices [I>2sigma(I)] } & \mathrm{R} 1=0.0625, \text { wR2 }=0.1476 \\ \text { R indices (all data) } & \mathrm{R} 1=0.0737, \mathrm{WR} 2=0.1541 \\ \text { Largest diff. peak and hole } & 1.019 \text { and }-0.488 \mathrm{e. \AA ^{-3 }}\end{array}$

Table S2. Atomic coordinates $\left(\times 10^{4}\right)$ and equivalent isotropic displacement parameters $\left(\AA^{2} \times 10^{3}\right)$ for 1. $U(\mathrm{eq})$ is defined as one third of the trace of the orthogonalized Uij tensor.

\begin{tabular}{|c|c|c|c|c|}
\hline & $\mathrm{x}$ & y & $\mathrm{z}$ & $\mathrm{U}(\mathrm{eq})$ \\
\hline Cl (2) & $5309(1)$ & $60(1)$ & $7899(1)$ & $61(1)$ \\
\hline Cl (1) & $4033(1)$ & $1583(1)$ & $7043(1)$ & $68(1)$ \\
\hline$C(13 A)$ & $10884(1)$ & $-931(2)$ & $5861(1)$ & $27(1)$ \\
\hline$C(1)$ & $9095(2)$ & $-3794(3)$ & $10321(1)$ & $40(1)$ \\
\hline$C(2)$ & $9364(2)$ & $-4530(3)$ & $10838(1)$ & $42(1)$ \\
\hline$C(3)$ & $10176(2)$ & $-4551(3)$ & $11185(1)$ & $39(1)$ \\
\hline$C(4)$ & $10690(2)$ & $-3845(3)$ & $10997(1)$ & $36(1)$ \\
\hline$C(4 A)$ & $10434(1)$ & $-3105(3)$ & $10442(1)$ & $29(1)$ \\
\hline$C(5)$ & $10949(1)$ & $-2434(3)$ & $10216(1)$ & $29(1)$ \\
\hline$C(5 A)$ & $10693(1)$ & $-1695(2)$ & $9679(1)$ & $26(1)$ \\
\hline$C(6)$ & $11232(1)$ & $-998(2)$ & $9455(1)$ & $26(1)$ \\
\hline$C(6 A)$ & $10949(1)$ & $-243(3)$ & $8938(1)$ & $28(1)$ \\
\hline$C(7)$ & $11474(1)$ & $541(3)$ & $8680(1)$ & $30(1)$ \\
\hline$C(7 A)$ & $11238(1)$ & $254(2)$ & $8014(1)$ & $26(1)$ \\
\hline$C(8)$ & 11755 (1) & $-136(2)$ & $7746(1)$ & $25(1)$ \\
\hline$C(8 A)$ & $11471(1)$ & $-379(2)$ & $7109(1)$ & $24(1)$ \\
\hline$C(9)$ & $11978(1)$ & $-592(2)$ & $6797(1)$ & $25(1)$ \\
\hline$C(9 A)$ & $11712(1)$ & $-852(2)$ & $6181(1)$ & $27(1)$ \\
\hline$C(10)$ & $12225(2)$ & $-1100(3)$ & $5862(1)$ & $33(1)$ \\
\hline$C(11)$ & $11946(2)$ & $-1447(3)$ & $5274(1)$ & $41(1)$ \\
\hline$C(12)$ & $11124(2)$ & $-1560(3)$ & $4958(1)$ & $39(1)$ \\
\hline$C(13)$ & $10615(2)$ & $-1310(3)$ & $5241(1)$ & $31(1)$ \\
\hline$C(14)$ & $10374(1)$ & $-651(2)$ & $6164(1)$ & $26(1)$ \\
\hline$C(14 A)$ & $10642(1)$ & $-349(2)$ & $6776(1)$ & $24(1)$ \\
\hline
\end{tabular}




\begin{tabular}{|c|c|c|c|c|}
\hline$C(15)$ & $10115(1)$ & $23(2)$ & $7082(1)$ & $24(1)$ \\
\hline$C(15 A)$ & $10411(1)$ & $348(2)$ & $7677(1)$ & $25(1)$ \\
\hline$C(16)$ & $9900(1)$ & $706(3)$ & $8037(1)$ & $28(1)$ \\
\hline$C(16 A)$ & $10125(1)$ & $-172(3)$ & $8600(1)$ & $28(1)$ \\
\hline C (17) & 9595 (1) & $-864(3)$ & $8783(1)$ & $29(1)$ \\
\hline$C(17 A)$ & 9867 ( 1) & $-1629(3)$ & $9339(1)$ & $27(1)$ \\
\hline $\mathrm{C}(18)$ & 9355 ( 1) & $-2332(3)$ & $9559(1)$ & $32(1)$ \\
\hline$C(18 A)$ & $9614(1)$ & $-3059(3)$ & $10099(1)$ & $30(1)$ \\
\hline C (19) & $12097(1)$ & $-1122(3)$ & $9785(1)$ & $29(1)$ \\
\hline$C(20)$ & $12506(2)$ & $-114(3)$ & $10190(1)$ & $36(1)$ \\
\hline C (21) & $13313(2)$ & $-208(3)$ & $10470(1)$ & $45(1)$ \\
\hline C (22) & $13715(2)$ & $-1301(4)$ & $10349(1)$ & $46(1)$ \\
\hline C (23) & $13316(2)$ & $-2317(4)$ & $9946(1)$ & $49(1)$ \\
\hline C (24) & $12509(2)$ & $-2229(3)$ & $9667(1)$ & $40(1)$ \\
\hline$C(25)$ & $12602(1)$ & $-385(2)$ & 8107 (1) & $25(1)$ \\
\hline$C(26)$ & 13088 (1) & $693(3)$ & $8410(1)$ & $36(1)$ \\
\hline C (27) & $13854(2)$ & $436(3)$ & $8779(1)$ & $43(1)$ \\
\hline C (28) & $14153(1)$ & $-892(3)$ & $8843(1)$ & $35(1)$ \\
\hline C (29) & $13683(1)$ & $-1973(3)$ & $8532(1)$ & $35(1)$ \\
\hline$C(30)$ & $12914(1)$ & $-1718(3)$ & $8164(1)$ & $31(1)$ \\
\hline C (31) & $9254(1)$ & $60(2)$ & $6737(1)$ & $25(1)$ \\
\hline C ( 32$)$ & $8850(1)$ & $-1167(3)$ & $6511(1)$ & $34(1)$ \\
\hline C (33) & $8054(2)$ & $-1142(3)$ & $6190(1)$ & $43(1)$ \\
\hline C (34) & $7650(2)$ & $108(3)$ & $6094(1)$ & $42(1)$ \\
\hline C (35) & 8041 (2) & $1330(3)$ & $6311(1)$ & $38(1)$ \\
\hline$C(36)$ & $8843(1)$ & 1307 (3) & $6630(1)$ & $33(1)$ \\
\hline C (37) & $8739(1)$ & $-869(3)$ & $8412(1)$ & $28(1)$ \\
\hline C (38) & 8447 (2) & $-1727(4)$ & 7918 (1) & $48(1)$ \\
\hline C (39) & $7659(2)$ & $-1726(4)$ & $7572(1)$ & $54(1)$ \\
\hline$C(40)$ & $7146(1)$ & $-882(3)$ & $7716(1)$ & $40(1)$ \\
\hline C ( 41$)$ & $7425(2)$ & $-30(3)$ & $8208(1)$ & $42(1)$ \\
\hline C ( 42) & $8218(2)$ & $-22(3)$ & $8558(1)$ & $37(1)$ \\
\hline C ( 43) & $4330(2)$ & $-4(5)$ & 7432 (2) & $67(1)$ \\
\hline
\end{tabular}

Table S3. Bond lengths $[\AA]$ and angles $\left[^{\circ}\right]$ for $\mathbf{1}$.

$\begin{array}{ll}C 1(2)-C(43) & 1.740(3) \\ C 1(1)-C(43) & 1.764(4) \\ C(13 A)-C(14) & 1.397(3) \\ C(13 A)-C(13) & 1.433(3) \\ C(13 A)-C(9 A) & 1.436(3) \\ C(1)-C(2) & 1.355(4) \\ C(1)-C(18 A) & 1.427(4) \\ C(1)-H(1) & 0.93(3) \\ C(2)-C(3) & 1.422(4) \\ C(2)-H(2) & 0.93(3) \\ C(3)-C(4) & 1.358(4) \\ C(3)-H(3) & 0.92(3) \\ C(4)-C(4 A) & 1.429(3) \\ C(4)-H(4) & 0.95(3) \\ C(4 A)-C(5) & 1.398(3) \\ C(4 A)-C(18 A) & 1.431(3)\end{array}$




\begin{tabular}{|c|c|}
\hline$C(5)-C(5 A)$ & $1.393(3)$ \\
\hline $\mathrm{C}(5)-\mathrm{H}(5)$ & $0.93(3)$ \\
\hline$C(5 A)-C(17 A)$ & $1.440(3)$ \\
\hline$C(5 A)-C(6)$ & $1.443(3)$ \\
\hline$C(6)-C(6 A)$ & $1.365(3)$ \\
\hline$C(6)-C(19)$ & $1.500(3)$ \\
\hline$C(6 A)-C(16 A)$ & $1.437(3)$ \\
\hline$C(6 A)-C(7)$ & $1.512(3)$ \\
\hline$C(7)-C(7 A)$ & $1.520(3)$ \\
\hline $\mathrm{C}(7)-\mathrm{H}(7 \mathrm{~A})$ & $0.96(2)$ \\
\hline $\mathrm{C}(7)-\mathrm{H}(7 \mathrm{~B})$ & $1.04(3)$ \\
\hline$C(7 A)-C(8)$ & $1.370(3)$ \\
\hline$C(7 A)-C(15 A)$ & $1.441(3)$ \\
\hline$C(8)-C(8 A)$ & $1.443(3)$ \\
\hline$C(8)-C(25)$ & $1.501(3)$ \\
\hline$C(8 A)-C(9)$ & $1.396(3)$ \\
\hline$C(8 A)-C(14 A)$ & $1.440(3)$ \\
\hline$C(9)-C(9 A)$ & $1.399(3)$ \\
\hline $\mathrm{C}(9)-\mathrm{H}(9)$ & $0.93(2)$ \\
\hline$C(9 A)-C(10)$ & $1.424(3)$ \\
\hline$C(10)-C(11)$ & $1.354(4)$ \\
\hline $\mathrm{C}(10)-\mathrm{H}(10)$ & $0.93(3)$ \\
\hline$C(11)-C(12)$ & $1.427(4)$ \\
\hline $\mathrm{C}(11)-\mathrm{H}(11)$ & $0.98(3)$ \\
\hline$C(12)-C(13)$ & $1.351(4)$ \\
\hline $\mathrm{C}(12)-\mathrm{H}(12)$ & $0.92(3)$ \\
\hline $\mathrm{C}(13)-\mathrm{H}(13)$ & $0.97(3)$ \\
\hline$C(14)-C(14 A)$ & $1.398(3)$ \\
\hline $\mathrm{C}(14)-\mathrm{H}(14)$ & $0.93(3)$ \\
\hline$C(14 A)-C(15)$ & $1.445(3)$ \\
\hline$C(15)-C(15 A)$ & $1.367(3)$ \\
\hline$C(15)-C(31)$ & $1.496(3)$ \\
\hline$C(15 A)-C(16)$ & $1.518(3)$ \\
\hline$C(16)-C(16 A)$ & $1.514(3)$ \\
\hline $\mathrm{C}(16)-\mathrm{H}(16 \mathrm{~A})$ & $0.98(3)$ \\
\hline $\mathrm{C}(16)-\mathrm{H}(16 \mathrm{~B})$ & $0.95(2)$ \\
\hline$C(16 A)-C(17)$ & $1.369(3)$ \\
\hline$C(17)-C(17 A)$ & $1.442(3)$ \\
\hline$C(17)-C(37)$ & $1.500(3)$ \\
\hline$C(17 A)-C(18)$ & $1.399(3)$ \\
\hline$C(18)-C(18 A)$ & $1.394(3)$ \\
\hline $\mathrm{C}(18)-\mathrm{H}(18)$ & $0.99(3)$ \\
\hline$C(19)-C(20)$ & $1.385(4)$ \\
\hline$C(19)-C(24)$ & $1.387(4)$ \\
\hline$C(20)-C(21)$ & $1.389(4)$ \\
\hline $\mathrm{C}(20)-\mathrm{H}(20)$ & $0.92(3)$ \\
\hline$C(21)-C(22)$ & $1.368(5)$ \\
\hline $\mathrm{C}(21)-\mathrm{H}(21)$ & $0.93(4)$ \\
\hline$C(22)-C(23)$ & $1.381(5)$ \\
\hline $\mathrm{C}(22)-\mathrm{H}(22)$ & $0.94(3)$ \\
\hline$C(23)-C(24)$ & $1.389(4)$ \\
\hline $\mathrm{C}(23)-\mathrm{H}(23)$ & $0.91(3)$ \\
\hline $\mathrm{C}(24)-\mathrm{H}(24)$ & $0.96(3)$ \\
\hline$C(25)-C(30)$ & $1.385(3)$ \\
\hline
\end{tabular}




\begin{tabular}{|c|c|}
\hline$C(25)-C(26)$ & $1.387(3)$ \\
\hline$C(26)-C(27)$ & $1.387(4)$ \\
\hline $\mathrm{C}(26)-\mathrm{H}(26)$ & $0.94(3)$ \\
\hline$C(27)-C(28)$ & $1.371(4)$ \\
\hline $\mathrm{C}(27)-\mathrm{H}(27)$ & $0.96(4)$ \\
\hline$C(28)-C(29)$ & $1.383(4)$ \\
\hline $\mathrm{C}(28)-\mathrm{H}(28)$ & $0.95(2)$ \\
\hline$C(29)-C(30)$ & $1.390(3)$ \\
\hline $\mathrm{C}(29)-\mathrm{H}(29)$ & $0.97(3)$ \\
\hline $\mathrm{C}(30)-\mathrm{H}(30)$ & $0.95(3)$ \\
\hline$C(31)-C(36)$ & $1.386(3)$ \\
\hline$C(31)-C(32)$ & $1.390(3)$ \\
\hline$C(32)-C(33)$ & $1.385(3)$ \\
\hline $\mathrm{C}(32)-\mathrm{H}(32)$ & $0.99(3)$ \\
\hline$C(33)-C(34)$ & $1.383(4)$ \\
\hline $\mathrm{C}(33)-\mathrm{H}(33)$ & $0.96(4)$ \\
\hline$C(34)-C(35)$ & $1.374(4)$ \\
\hline $\mathrm{C}(34)-\mathrm{H}(34)$ & $0.87(3)$ \\
\hline$C(35)-C(36)$ & $1.392(3)$ \\
\hline $\mathrm{C}(35)-\mathrm{H}(35)$ & $0.90(3)$ \\
\hline $\mathrm{C}(36)-\mathrm{H}(36)$ & $0.92(3)$ \\
\hline$C(37)-C(38)$ & $1.381(4)$ \\
\hline$C(37)-C(42)$ & $1.388(3)$ \\
\hline$C(38)-C(39)$ & $1.383(4)$ \\
\hline $\mathrm{C}(38)-\mathrm{H}(38)$ & $0.94(4)$ \\
\hline$C(39)-C(40)$ & $1.371(4)$ \\
\hline $\mathrm{C}(39)-\mathrm{H}(39)$ & $0.91(4)$ \\
\hline$C(40)-C(41)$ & $1.373(4)$ \\
\hline $\mathrm{C}(40)-\mathrm{H}(40)$ & $0.94(3)$ \\
\hline$C(41)-C(42)$ & $1.392(4)$ \\
\hline $\mathrm{C}(41)-\mathrm{H}(41)$ & $0.94(3)$ \\
\hline $\mathrm{C}(42)-\mathrm{H}(42)$ & $0.96(3)$ \\
\hline $\mathrm{C}(43)-\mathrm{H}(43 \mathrm{~A})$ & 0.9900 \\
\hline $\mathrm{C}(43)-\mathrm{H}(43 \mathrm{~B})$ & 0.9900 \\
\hline$C(14)-C(13 A)-C(13)$ & $122.6(2)$ \\
\hline$C(14)-C(13 A)-C(9 A)$ & $119.1(2)$ \\
\hline$C(13)-C(13 A)-C(9 A)$ & $118.3(2)$ \\
\hline$C(2)-C(1)-C(18 A)$ & $121.5(2)$ \\
\hline $\mathrm{C}(2)-\mathrm{C}(1)-\mathrm{H}(1)$ & $121(2)$ \\
\hline $\mathrm{C}(18 \mathrm{~A})-\mathrm{C}(1)-\mathrm{H}(1)$ & $117.8(19)$ \\
\hline$C(1)-C(2)-C(3)$ & $120.3(3)$ \\
\hline $\mathrm{C}(1)-\mathrm{C}(2)-\mathrm{H}(2)$ & $122.7(19)$ \\
\hline $\mathrm{C}(3)-\mathrm{C}(2)-\mathrm{H}(2)$ & $116.9(19)$ \\
\hline$C(4)-C(3)-C(2)$ & $120.1(3)$ \\
\hline $\mathrm{C}(4)-\mathrm{C}(3)-\mathrm{H}(3)$ & $119.6(18)$ \\
\hline $\mathrm{C}(2)-\mathrm{C}(3)-\mathrm{H}(3)$ & $120.2(18)$ \\
\hline$C(3)-C(4)-C(4 A)$ & $121.2(2)$ \\
\hline $\mathrm{C}(3)-\mathrm{C}(4)-\mathrm{H}(4)$ & $121.7(17)$ \\
\hline$C(4 A)-C(4)-H(4)$ & $117.1(17)$ \\
\hline$C(5)-C(4 A)-C(4)$ & $123.0(2)$ \\
\hline$C(5)-C(4 A)-C(18 A)$ & $118.5(2)$ \\
\hline$C(4)-C(4 A)-C(18 A)$ & $118.6(2)$ \\
\hline$C(5 A)-C(5)-C(4 A)$ & $122.6(2)$ \\
\hline
\end{tabular}




\begin{tabular}{|c|c|}
\hline$C(5 A)-C(5)-H(5)$ & $119.8(16)$ \\
\hline$C(4 A)-C(5)-H(5)$ & $117.6(15)$ \\
\hline$C(5)-C(5 A)-C(17 A)$ & $119.0(2)$ \\
\hline$C(5)-C(5 A)-C(6)$ & $121.9(2)$ \\
\hline$C(17 A)-C(5 A)-C(6)$ & $119.2(2)$ \\
\hline$C(6 A)-C(6)-C(5 A)$ & $119.5(2)$ \\
\hline$C(6 A)-C(6)-C(19)$ & $120.5(2)$ \\
\hline$C(5 A)-C(6)-C(19)$ & $120.0(2)$ \\
\hline$C(6)-C(6 A)-C(16 A)$ & $121.3(2)$ \\
\hline$C(6)-C(6 A)-C(7)$ & $122.7(2)$ \\
\hline$C(16 A)-C(6 A)-C(7)$ & $116.0(2)$ \\
\hline$C(6 A)-C(7)-C(7 A)$ & $110.55(19)$ \\
\hline$C(6 A)-C(7)-H(7 A)$ & $110.9(15)$ \\
\hline$C(7 A)-C(7)-H(7 A)$ & $112.2(14)$ \\
\hline$C(6 A)-C(7)-H(7 B)$ & $109.2(14)$ \\
\hline$C(7 A)-C(7)-H(7 B)$ & $109.8(14)$ \\
\hline $\mathrm{H}(7 \mathrm{~A})-\mathrm{C}(7)-\mathrm{H}(7 \mathrm{~B})$ & $104(2)$ \\
\hline$C(8)-C(7 A)-C(15 A)$ & $121.1(2)$ \\
\hline$C(8)-C(7 A)-C(7)$ & $123.6(2)$ \\
\hline$C(15 A)-C(7 A)-C(7)$ & $115.22(19)$ \\
\hline$C(7 A)-C(8)-C(8 A)$ & $119.3(2)$ \\
\hline$C(7 A)-C(8)-C(25)$ & $121.2(2)$ \\
\hline$C(8 A)-C(8)-C(25)$ & $119.47(19)$ \\
\hline$C(9)-C(8 A)-C(14 A)$ & $118.4(2)$ \\
\hline$C(9)-C(8 A)-C(8)$ & $122.0(2)$ \\
\hline$C(14 A)-C(8 A)-C(8)$ & $119.6(2)$ \\
\hline$C(8 A)-C(9)-C(9 A)$ & $122.7(2)$ \\
\hline$C(8 A)-C(9)-H(9)$ & $119.6(15)$ \\
\hline$C(9 A)-C(9)-H(9)$ & $117.6(15)$ \\
\hline$C(9)-C(9 A)-C(10)$ & $123.1(2)$ \\
\hline$C(9)-C(9 A)-C(13 A)$ & $118.4(2)$ \\
\hline$C(10)-C(9 A)-C(13 A)$ & $118.4(2)$ \\
\hline$C(11)-C(10)-C(9 A)$ & $121.4(2)$ \\
\hline $\mathrm{C}(11)-\mathrm{C}(10)-\mathrm{H}(10)$ & $121.6(15)$ \\
\hline $\mathrm{C}(9 \mathrm{~A})-\mathrm{C}(10)-\mathrm{H}(10)$ & $117.1(15)$ \\
\hline$C(10)-C(11)-C(12)$ & $120.4(2)$ \\
\hline $\mathrm{C}(10)-\mathrm{C}(11)-\mathrm{H}(11)$ & $122.1(17)$ \\
\hline $\mathrm{C}(12)-\mathrm{C}(11)-\mathrm{H}(11)$ & $117.5(17)$ \\
\hline$C(13)-C(12)-C(11)$ & $120.3(2)$ \\
\hline $\mathrm{C}(13)-\mathrm{C}(12)-\mathrm{H}(12)$ & $120.1(18)$ \\
\hline $\mathrm{C}(11)-\mathrm{C}(12)-\mathrm{H}(12)$ & $119.6(17)$ \\
\hline$C(12)-C(13)-C(13 A)$ & $121.2(2)$ \\
\hline $\mathrm{C}(12)-\mathrm{C}(13)-\mathrm{H}(13)$ & $123.6(17)$ \\
\hline$C(13 A)-C(13)-H(13)$ & $115.1(17)$ \\
\hline$C(13 A)-C(14)-C(14 A)$ & $122.2(2)$ \\
\hline$C(13 A)-C(14)-H(14)$ & $118.4(16)$ \\
\hline$C(14 A)-C(14)-H(14)$ & $119.4(16)$ \\
\hline$C(14)-C(14 A)-C(8 A)$ & $118.9(2)$ \\
\hline$C(14)-C(14 A)-C(15)$ & $122.1(2)$ \\
\hline$C(8 A)-C(14 A)-C(15)$ & $119.04(19)$ \\
\hline$C(15 A)-C(15)-C(14 A)$ & $119.61(19)$ \\
\hline$C(15 A)-C(15)-C(31)$ & $121.2(2)$ \\
\hline$C(14 A)-C(15)-C(31)$ & $119.16(19)$ \\
\hline$C(15)-C(15 A)-C(7 A)$ & $121.1(2)$ \\
\hline
\end{tabular}




\begin{tabular}{|c|c|}
\hline$C(15)-C(15 A)-C(16)$ & $123.1(2)$ \\
\hline$C(7 A)-C(15 A)-C(16)$ & $115.59(19)$ \\
\hline$C(16 A)-C(16)-C(15 A)$ & $110.53(19)$ \\
\hline$C(16 A)-C(16)-H(16 A)$ & $109.1(14)$ \\
\hline$C(15 A)-C(16)-H(16 A)$ & $108.5(14)$ \\
\hline$C(16 A)-C(16)-H(16 B)$ & $109.8(15)$ \\
\hline$C(15 A)-C(16)-H(16 B)$ & $112.2(15)$ \\
\hline $\mathrm{H}(16 \mathrm{~A})-\mathrm{C}(16)-\mathrm{H}(16 \mathrm{~B})$ & $106(2)$ \\
\hline$C(17)-C(16 A)-C(6 A)$ & $121.1(2)$ \\
\hline$C(17)-C(16 A)-C(16)$ & $123.7(2)$ \\
\hline$C(6 A)-C(16 A)-C(16)$ & $115.2(2)$ \\
\hline$C(16 A)-C(17)-C(17 A)$ & $119.4(2)$ \\
\hline$C(16 A)-C(17)-C(37)$ & $121.3(2)$ \\
\hline$C(17 A)-C(17)-C(37)$ & $119.3(2)$ \\
\hline$C(18)-C(17 A)-C(5 A)$ & $118.3(2)$ \\
\hline$C(18)-C(17 A)-C(17)$ & $122.2(2)$ \\
\hline$C(5 A)-C(17 A)-C(17)$ & $119.5(2)$ \\
\hline$C(18 A)-C(18)-C(17 A)$ & $122.4(2)$ \\
\hline $\mathrm{C}(18 \mathrm{~A})-\mathrm{C}(18)-\mathrm{H}(18)$ & $119.7(15)$ \\
\hline$C(17 A)-C(18)-H(18)$ & $117.9(15)$ \\
\hline$C(18)-C(18 A)-C(1)$ & $122.6(2)$ \\
\hline$C(18)-C(18 A)-C(4 A)$ & $119.2(2)$ \\
\hline$C(1)-C(18 A)-C(4 A)$ & $118.1(2)$ \\
\hline$C(20)-C(19)-C(24)$ & $118.6(2)$ \\
\hline$C(20)-C(19)-C(6)$ & $121.2(2)$ \\
\hline$C(24)-C(19)-C(6)$ & $120.1(2)$ \\
\hline$C(19)-C(20)-C(21)$ & $120.5(3)$ \\
\hline $\mathrm{C}(19)-\mathrm{C}(20)-\mathrm{H}(20)$ & $119.1(17)$ \\
\hline$C(21)-C(20)-H(20)$ & $120.3(17)$ \\
\hline$C(22)-C(21)-C(20)$ & $120.4(3)$ \\
\hline $\mathrm{C}(22)-\mathrm{C}(21)-\mathrm{H}(21)$ & $120(2)$ \\
\hline $\mathrm{C}(20)-\mathrm{C}(21)-\mathrm{H}(21)$ & $119(2)$ \\
\hline$C(21)-C(22)-C(23)$ & $119.8(3)$ \\
\hline $\mathrm{C}(21)-\mathrm{C}(22)-\mathrm{H}(22)$ & $121.9(19)$ \\
\hline$C(23)-C(22)-H(22)$ & $118.3(19)$ \\
\hline$C(22)-C(23)-C(24)$ & $120.0(3)$ \\
\hline$C(22)-C(23)-H(23)$ & $121(2)$ \\
\hline $\mathrm{C}(24)-\mathrm{C}(23)-\mathrm{H}(23)$ & $119(2)$ \\
\hline$C(19)-C(24)-C(23)$ & $120.7(3)$ \\
\hline $\mathrm{C}(19)-\mathrm{C}(24)-\mathrm{H}(24)$ & $120.1(18)$ \\
\hline $\mathrm{C}(23)-\mathrm{C}(24)-\mathrm{H}(24)$ & $119.2(18)$ \\
\hline$C(30)-C(25)-C(26)$ & $118.1(2)$ \\
\hline$C(30)-C(25)-C(8)$ & $120.5(2)$ \\
\hline$C(26)-C(25)-C(8)$ & $121.4(2)$ \\
\hline$C(27)-C(26)-C(25)$ & $120.9(2)$ \\
\hline $\mathrm{C}(27)-\mathrm{C}(26)-\mathrm{H}(26)$ & $118.7(18)$ \\
\hline $\mathrm{C}(25)-\mathrm{C}(26)-\mathrm{H}(26)$ & $120.4(17)$ \\
\hline$C(28)-C(27)-C(26)$ & $120.6(2)$ \\
\hline $\mathrm{C}(28)-\mathrm{C}(27)-\mathrm{H}(27)$ & $120(2)$ \\
\hline $\mathrm{C}(26)-\mathrm{C}(27)-\mathrm{H}(27)$ & $119(2)$ \\
\hline$C(27)-C(28)-C(29)$ & $119.2(2)$ \\
\hline $\mathrm{C}(27)-\mathrm{C}(28)-\mathrm{H}(28)$ & $121.4(15)$ \\
\hline $\mathrm{C}(29)-\mathrm{C}(28)-\mathrm{H}(28)$ & $119.4(15)$ \\
\hline$C(28)-C(29)-C(30)$ & $120.3(2)$ \\
\hline
\end{tabular}




\begin{tabular}{|c|c|}
\hline $\mathrm{C}(28)-\mathrm{C}(29)-\mathrm{H}(29)$ & $121.0(16)$ \\
\hline $\mathrm{C}(30)-\mathrm{C}(29)-\mathrm{H}(29)$ & $118.7(16)$ \\
\hline$C(25)-C(30)-C(29)$ & $120.9(2)$ \\
\hline $\mathrm{C}(25)-\mathrm{C}(30)-\mathrm{H}(30)$ & $121.8(15)$ \\
\hline $\mathrm{C}(29)-\mathrm{C}(30)-\mathrm{H}(30)$ & $117.2(15)$ \\
\hline$C(36)-C(31)-C(32)$ & $118.7(2)$ \\
\hline$C(36)-C(31)-C(15)$ & $121.1(2)$ \\
\hline$C(32)-C(31)-C(15)$ & $120.2(2)$ \\
\hline$C(33)-C(32)-C(31)$ & $120.6(2)$ \\
\hline $\mathrm{C}(33)-\mathrm{C}(32)-\mathrm{H}(32)$ & $118.2(16)$ \\
\hline $\mathrm{C}(31)-\mathrm{C}(32)-\mathrm{H}(32)$ & $121.2(16)$ \\
\hline$C(34)-C(33)-C(32)$ & $120.1(3)$ \\
\hline $\mathrm{C}(34)-\mathrm{C}(33)-\mathrm{H}(33)$ & $122(2)$ \\
\hline $\mathrm{C}(32)-\mathrm{C}(33)-\mathrm{H}(33)$ & $118(2)$ \\
\hline$C(35)-C(34)-C(33)$ & $120.0(2)$ \\
\hline $\mathrm{C}(35)-\mathrm{C}(34)-\mathrm{H}(34)$ & $121(2)$ \\
\hline $\mathrm{C}(33)-\mathrm{C}(34)-\mathrm{H}(34)$ & $119(2)$ \\
\hline$C(34)-C(35)-C(36)$ & $120.0(3)$ \\
\hline $\mathrm{C}(34)-\mathrm{C}(35)-\mathrm{H}(35)$ & $122.1(19)$ \\
\hline $\mathrm{C}(36)-\mathrm{C}(35)-\mathrm{H}(35)$ & $117.9(19)$ \\
\hline$C(31)-C(36)-C(35)$ & $120.6(3)$ \\
\hline $\mathrm{C}(31)-\mathrm{C}(36)-\mathrm{H}(36)$ & $120.2(17)$ \\
\hline $\mathrm{C}(35)-\mathrm{C}(36)-\mathrm{H}(36)$ & $119.1(17)$ \\
\hline$C(38)-C(37)-C(42)$ & $118.2(2)$ \\
\hline$C(38)-C(37)-C(17)$ & $120.8(2)$ \\
\hline$C(42)-C(37)-C(17)$ & $121.0(2)$ \\
\hline$C(37)-C(38)-C(39)$ & $121.0(3)$ \\
\hline $\mathrm{C}(37)-\mathrm{C}(38)-\mathrm{H}(38)$ & $117(2)$ \\
\hline $\mathrm{C}(39)-\mathrm{C}(38)-\mathrm{H}(38)$ & $122(2)$ \\
\hline$C(40)-C(39)-C(38)$ & $120.7(3)$ \\
\hline $\mathrm{C}(40)-\mathrm{C}(39)-\mathrm{H}(39)$ & $119(2)$ \\
\hline $\mathrm{C}(38)-\mathrm{C}(39)-\mathrm{H}(39)$ & $121(2)$ \\
\hline$C(39)-C(40)-C(41)$ & $119.1(2)$ \\
\hline $\mathrm{C}(39)-\mathrm{C}(40)-\mathrm{H}(40)$ & $121.0(18)$ \\
\hline $\mathrm{C}(41)-\mathrm{C}(40)-\mathrm{H}(40)$ & $119.9(18)$ \\
\hline$C(40)-C(41)-C(42)$ & $120.6(3)$ \\
\hline $\mathrm{C}(40)-\mathrm{C}(41)-\mathrm{H}(41)$ & $119.5(19)$ \\
\hline $\mathrm{C}(42)-\mathrm{C}(41)-\mathrm{H}(41)$ & $119.9(19)$ \\
\hline$C(37)-C(42)-C(41)$ & $120.4(2)$ \\
\hline $\mathrm{C}(37)-\mathrm{C}(42)-\mathrm{H}(42)$ & $118.3(18)$ \\
\hline $\mathrm{C}(41)-\mathrm{C}(42)-\mathrm{H}(42)$ & $121.3(18)$ \\
\hline $\mathrm{Cl}(2)-\mathrm{C}(43)-\mathrm{Cl}(1)$ & $111.2(2)$ \\
\hline $\mathrm{Cl}(2)-\mathrm{C}(43)-\mathrm{H}(43 \mathrm{~A})$ & 109.4 \\
\hline $\mathrm{Cl}(1)-\mathrm{C}(43)-\mathrm{H}(43 \mathrm{~A})$ & 109.4 \\
\hline $\mathrm{Cl}(2)-\mathrm{C}(43)-\mathrm{H}(43 \mathrm{~B})$ & 109.4 \\
\hline $\mathrm{Cl}(1)-\mathrm{C}(43)-\mathrm{H}(43 \mathrm{~B})$ & 109.4 \\
\hline $\mathrm{H}(43 \mathrm{~A})-\mathrm{C}(43)-\mathrm{H}(43 \mathrm{~B})$ & 108.0 \\
\hline
\end{tabular}


Table S4. Anisotropic displacement parameters $\left(\AA^{2} \times 10^{3}\right)$ for 1 . The anisotropic displacement factor exponent takes the form: $-2 \mathrm{pi}^{2}\left[\mathrm{~h}^{2} \mathrm{a}^{2} \mathrm{U} 11+\ldots+2 \mathrm{~h} \mathrm{k} \mathrm{a} \mathrm{b}^{*} \mathrm{U} 12\right]$

\begin{tabular}{|c|c|c|c|c|c|c|}
\hline & $\mathrm{U} 11$ & U22 & U33 & U23 & U13 & $\mathrm{U} 12$ \\
\hline $\mathrm{Cl}(2)$ & $34(1)$ & $76(1)$ & $68(1)$ & $7(1)$ & $12(1)$ & $8(1)$ \\
\hline $\mathrm{Cl}(1)$ & $72(1)$ & $58(1)$ & $65(1)$ & $1(1)$ & $14(1)$ & $29(1)$ \\
\hline$C(13 A)$ & $30(1)$ & $24(1)$ & $27(1)$ & $4(1)$ & $10(1)$ & $3(1)$ \\
\hline$C(1)$ & $27(1)$ & $61(2)$ & $32(1)$ & $-3(1)$ & $8(1)$ & $-9(1)$ \\
\hline$C(2)$ & $37(1)$ & $60(2)$ & $33(1)$ & $-2(1)$ & $18(1)$ & $-13(1)$ \\
\hline$C(3)$ & $38(1)$ & $53(2)$ & $29(1)$ & $4(1)$ & $14(1)$ & $2(1)$ \\
\hline$C(4)$ & $26(1)$ & $49(2)$ & $30(1)$ & $1(1)$ & $9(1)$ & $2(1)$ \\
\hline$C(4 A)$ & $25(1)$ & $37(1)$ & $25(1)$ & $-5(1)$ & $9(1)$ & $1(1)$ \\
\hline$C(5)$ & $18(1)$ & $38(1)$ & $26(1)$ & $-4(1)$ & $5(1)$ & $-1(1)$ \\
\hline$C(5 A)$ & $20(1)$ & $33(1)$ & $24(1)$ & $-6(1)$ & $7(1)$ & $-1(1)$ \\
\hline$C(6)$ & $20(1)$ & $34(1)$ & $23(1)$ & $-4(1)$ & $5(1)$ & $0(1)$ \\
\hline$C(6 A)$ & $23(1)$ & $34(1)$ & $25(1)$ & $-6(1)$ & $8(1)$ & $-2(1)$ \\
\hline$C(7)$ & $23(1)$ & $40(1)$ & $23(1)$ & $-2(1)$ & $6(1)$ & $-3(1)$ \\
\hline$C(7 A)$ & $23(1)$ & $28(1)$ & $24(1)$ & $1(1)$ & $6(1)$ & $-2(1)$ \\
\hline $\mathrm{C}(8)$ & $22(1)$ & $21(1)$ & $29(1)$ & $1(1)$ & $7(1)$ & $-2(1)$ \\
\hline$C(8 A)$ & $23(1)$ & $19(1)$ & $27(1)$ & $2(1)$ & $7(1)$ & $0(1)$ \\
\hline$C(9)$ & $20(1)$ & $24(1)$ & $31(1)$ & $4(1)$ & $7(1)$ & $1(1)$ \\
\hline$C(9 A)$ & $28(1)$ & $23(1)$ & $31(1)$ & $4(1)$ & $12(1)$ & $2(1)$ \\
\hline$C(10)$ & $28(1)$ & $39(1)$ & $36(1)$ & $6(1)$ & $14(1)$ & $5(1)$ \\
\hline C (11) & $40(2)$ & $52(2)$ & $37(1)$ & $4(1)$ & $23(1)$ & $11(1)$ \\
\hline$C(12)$ & $46(2)$ & $45(2)$ & $26(1)$ & $1(1)$ & $15(1)$ & $8(1)$ \\
\hline C (13) & $32(1)$ & $33(1)$ & $28(1)$ & $2(1)$ & $10(1)$ & $4(1)$ \\
\hline$C(14)$ & $23(1)$ & $27(1)$ & $25(1)$ & $2(1)$ & $6(1)$ & $2(1)$ \\
\hline$C(14 A)$ & $23(1)$ & $21(1)$ & $26(1)$ & $3(1)$ & $8(1)$ & $0(1)$ \\
\hline C (15) & $21(1)$ & $23(1)$ & $26(1)$ & $2(1)$ & $7(1)$ & $1(1)$ \\
\hline$C(15 A)$ & $23(1)$ & $27(1)$ & $26(1)$ & $0(1)$ & $9(1)$ & $1(1)$ \\
\hline$C(16)$ & $23(1)$ & $35(1)$ & $25(1)$ & $-1(1)$ & $7(1)$ & $3(1)$ \\
\hline$C(16 A)$ & $23(1)$ & $36(1)$ & $24(1)$ & $-4(1)$ & $7(1)$ & $2(1)$ \\
\hline C (17) & $21(1)$ & $39(1)$ & $25(1)$ & $-6(1)$ & $6(1)$ & $1(1)$ \\
\hline$C(17 A)$ & $22(1)$ & $35(1)$ & $24(1)$ & $-6(1)$ & $7(1)$ & $-1(1)$ \\
\hline$C(18)$ & $20(1)$ & $45(2)$ & $29(1)$ & $-6(1)$ & $6(1)$ & $-3(1)$ \\
\hline$C(18 A)$ & $24(1)$ & $42(1)$ & $24(1)$ & $-6(1)$ & $9(1)$ & $-4(1)$ \\
\hline C (19) & $20(1)$ & $42(1)$ & $23(1)$ & $4(1)$ & $6(1)$ & $-2(1)$ \\
\hline C (20) & $30(1)$ & $41(2)$ & $32(1)$ & $-2(1)$ & $5(1)$ & $-4(1)$ \\
\hline C (21) & $32(1)$ & $58(2)$ & $37(1)$ & $4(1)$ & $-1(1)$ & $-17(1)$ \\
\hline C (22) & $19(1)$ & $70(2)$ & $43(2)$ & $15(1)$ & $3(1)$ & $-4(1)$ \\
\hline C (23) & $28(1)$ & $67(2)$ & $49(2)$ & $5(2)$ & $11(1)$ & $12(1)$ \\
\hline C (24) & $26(1)$ & $52(2)$ & $38(1)$ & $-5(1)$ & $7(1)$ & $2(1)$ \\
\hline$C(25)$ & $21(1)$ & $29(1)$ & $24(1)$ & $2(1)$ & $8(1)$ & $-2(1)$ \\
\hline$C(26)$ & $28(1)$ & $23(1)$ & $51(2)$ & $-4(1)$ & $7(1)$ & $0(1)$ \\
\hline C (27) & $26(1)$ & $34(1)$ & $57(2)$ & $-12(1)$ & $1(1)$ & $-6(1)$ \\
\hline C (28) & $21(1)$ & $43(2)$ & $34(1)$ & $-1(1)$ & $2(1)$ & $1(1)$ \\
\hline C (29) & $30(1)$ & $28(1)$ & $42(1)$ & $2(1)$ & $7(1)$ & $6(1)$ \\
\hline$C(30)$ & $27(1)$ & $26(1)$ & $35(1)$ & $-3(1)$ & $5(1)$ & $-3(1)$ \\
\hline$C(31)$ & $22(1)$ & $34(1)$ & $20(1)$ & $0(1)$ & $8(1)$ & $3(1)$ \\
\hline
\end{tabular}




\begin{tabular}{lrrrrrr}
$C(32)$ & $27(1)$ & $37(1)$ & $34(1)$ & $1(1)$ & $4(1)$ & $0(1)$ \\
$C(33)$ & $28(1)$ & $51(2)$ & $39(1)$ & $2(1)$ & $1(1)$ & $-8(1)$ \\
$C(34)$ & $20(1)$ & $70(2)$ & $30(1)$ & $11(1)$ & $2(1)$ & $2(1)$ \\
$C(35)$ & $31(1)$ & $51(2)$ & $31(1)$ & $6(1)$ & $10(1)$ & $17(1)$ \\
$C(36)$ & $31(1)$ & $36(1)$ & $30(1)$ & $-2(1)$ & $9(1)$ & $4(1)$ \\
$C(37)$ & $22(1)$ & $38(1)$ & $23(1)$ & $2(1)$ & $7(1)$ & $-1(1)$ \\
$C(38)$ & $28(1)$ & $68(2)$ & $44(2)$ & $-22(2)$ & $9(1)$ & $4(1)$ \\
$C(39)$ & $31(2)$ & $86(2)$ & $38(2)$ & $-27(2)$ & $4(1)$ & $-5(2)$ \\
$C(40)$ & $19(1)$ & $65(2)$ & $32(1)$ & $4(1)$ & $3(1)$ & $-1(1)$ \\
$C(41)$ & $25(1)$ & $52(2)$ & $50(2)$ & $1(1)$ & $13(1)$ & $8(1)$ \\
$C(42)$ & $29(1)$ & $45(2)$ & $37(1)$ & $-11(1)$ & $9(1)$ & $0(1)$ \\
$C(43)$ & $39(2)$ & $101(3)$ & $57(2)$ & $16(2)$ & $11(2)$ & $-3(2)$ \\
\hline
\end{tabular}


Table S5. Hydrogen coordinates $\left(\times 10^{4}\right)$ and isotropic displacement parameters $\left(\mathrm{A}^{2} \times 10^{3}\right)$ for $\mathbf{1}$.

\begin{tabular}{|c|c|c|c|c|}
\hline & $\mathrm{x}$ & y & z & $\mathrm{U}(\mathrm{eq})$ \\
\hline $\mathrm{H}(43 \mathrm{~A})$ & 4001 & -197 & 7674 & 81 \\
\hline $\mathrm{H}(43 \mathrm{~B})$ & 4254 & -774 & 7141 & 81 \\
\hline $\mathrm{H}(1)$ & $8564(19)$ & $-3790(30)$ & $10089(14)$ & $52(9)$ \\
\hline $\mathrm{H}(2)$ & $9037(18)$ & $-5050(30)$ & $10980(13)$ & $48(8)$ \\
\hline $\mathrm{H}(3)$ & $10360(16)$ & $-5080(30)$ & $11530(13)$ & $40(8)$ \\
\hline $\mathrm{H}(4)$ & $11234(17)$ & $-3820(30)$ & $11227(12)$ & $38(7)$ \\
\hline $\mathrm{H}(5)$ & $11479(15)$ & $-2490(30)$ & $10437(11)$ & $28(6)$ \\
\hline $\mathrm{H}(7 \mathrm{~A})$ & 12015 (15) & $330(30)$ & $8896(11)$ & $24(6)$ \\
\hline $\mathrm{H}(7 \mathrm{~B})$ & $11434(14)$ & $1610(30)$ & $8752(11)$ & $28(6)$ \\
\hline $\mathrm{H}(9)$ & 12517 (15) & $-560(30)$ & $7002(11)$ & $25(6)$ \\
\hline $\mathrm{H}(10)$ & $12759(15)$ & $-1040(30)$ & $6076(11)$ & $28(6)$ \\
\hline $\mathrm{H}(11)$ & $12292(17)$ & $-1670(30)$ & $5056(13)$ & $46(8)$ \\
\hline $\mathrm{H}(12)$ & $10942(16)$ & $-1810(30)$ & $4561(13)$ & $41(8)$ \\
\hline $\mathrm{H}(13)$ & $10050(17)$ & $-1330(30)$ & $5042(13)$ & $42(8)$ \\
\hline $\mathrm{H}(14)$ & $9839(16)$ & $-670(30)$ & $5949(11)$ & $32(7)$ \\
\hline $\mathrm{H}(16 \mathrm{~A})$ & 9975 (14) & $1700(30)$ & $8148(11)$ & $27(6)$ \\
\hline $\mathrm{H}(16 \mathrm{~B})$ & $9358(15)$ & $590(30)$ & $7810(11)$ & $25(6)$ \\
\hline $\mathrm{H}(18)$ & $8793(16)$ & $-2320(30)$ & 9315 (12) & $35(7)$ \\
\hline $\mathrm{H}(20)$ & $12239(15)$ & $630(30)$ & $10264(12)$ & $33(7)$ \\
\hline $\mathrm{H}(21)$ & $13580(20)$ & $480(40)$ & $10741(16)$ & $63(10)$ \\
\hline $\mathrm{H}(22)$ & $14258(18)$ & $-1390(30)$ & $10534(13)$ & $49(8)$ \\
\hline $\mathrm{H}(23)$ & $13573(18)$ & $-3050(40)$ & $9857(14)$ & $53(9)$ \\
\hline $\mathrm{H}(24)$ & $12239(17)$ & $-2940(30)$ & $9388(13)$ & $45(8)$ \\
\hline $\mathrm{H}(26)$ & $12899(16)$ & $1610(30)$ & 8375 (12) & $43(8)$ \\
\hline $\mathrm{H}(27)$ & $14172(19)$ & $1190(40)$ & $8992(15)$ & $61(9)$ \\
\hline $\mathrm{H}(28)$ & $14672(15)$ & $-1080(30)$ & $9098(11)$ & $26(6)$ \\
\hline $\mathrm{H}(29)$ & $13884(15)$ & $-2920(30)$ & $8558(12)$ & $35(7)$ \\
\hline $\mathrm{H}(30)$ & $12606(15)$ & $-2500(30)$ & $7972(11)$ & $30(6)$ \\
\hline $\mathrm{H}(32)$ & $9123(16)$ & $-2080(30)$ & $6570(12)$ & $40(7)$ \\
\hline $\mathrm{H}(33)$ & 7795 (19) & $-2020(40)$ & $6051(14)$ & $60(10)$ \\
\hline $\mathrm{H}(34)$ & $7150(20)$ & $120(40)$ & $5873(15)$ & $61(10)$ \\
\hline $\mathrm{H}(35)$ & $7796(17)$ & $2160(30)$ & $6256(13)$ & $43(8)$ \\
\hline $\mathrm{H}(36)$ & $9102(16)$ & $2140(30)$ & $6763(12)$ & $38(7)$ \\
\hline $\mathrm{H}(38)$ & $8800(20)$ & $-2310(40)$ & 7831 (15) & $65(10)$ \\
\hline $\mathrm{H}(39)$ & $7480(20)$ & $-2260(40)$ & $7237(16)$ & $67(10)$ \\
\hline $\mathrm{H}(40)$ & 6607 (17) & $-900(30)$ & 7487 (13) & $46(8)$ \\
\hline $\mathrm{H}(41)$ & $7076(18)$ & $550(30)$ & $8309(13)$ & $51(8)$ \\
\hline $\mathrm{H}(42)$ & 8421 (17) & $590(30)$ & $8895(14)$ & $47(8)$ \\
\hline
\end{tabular}




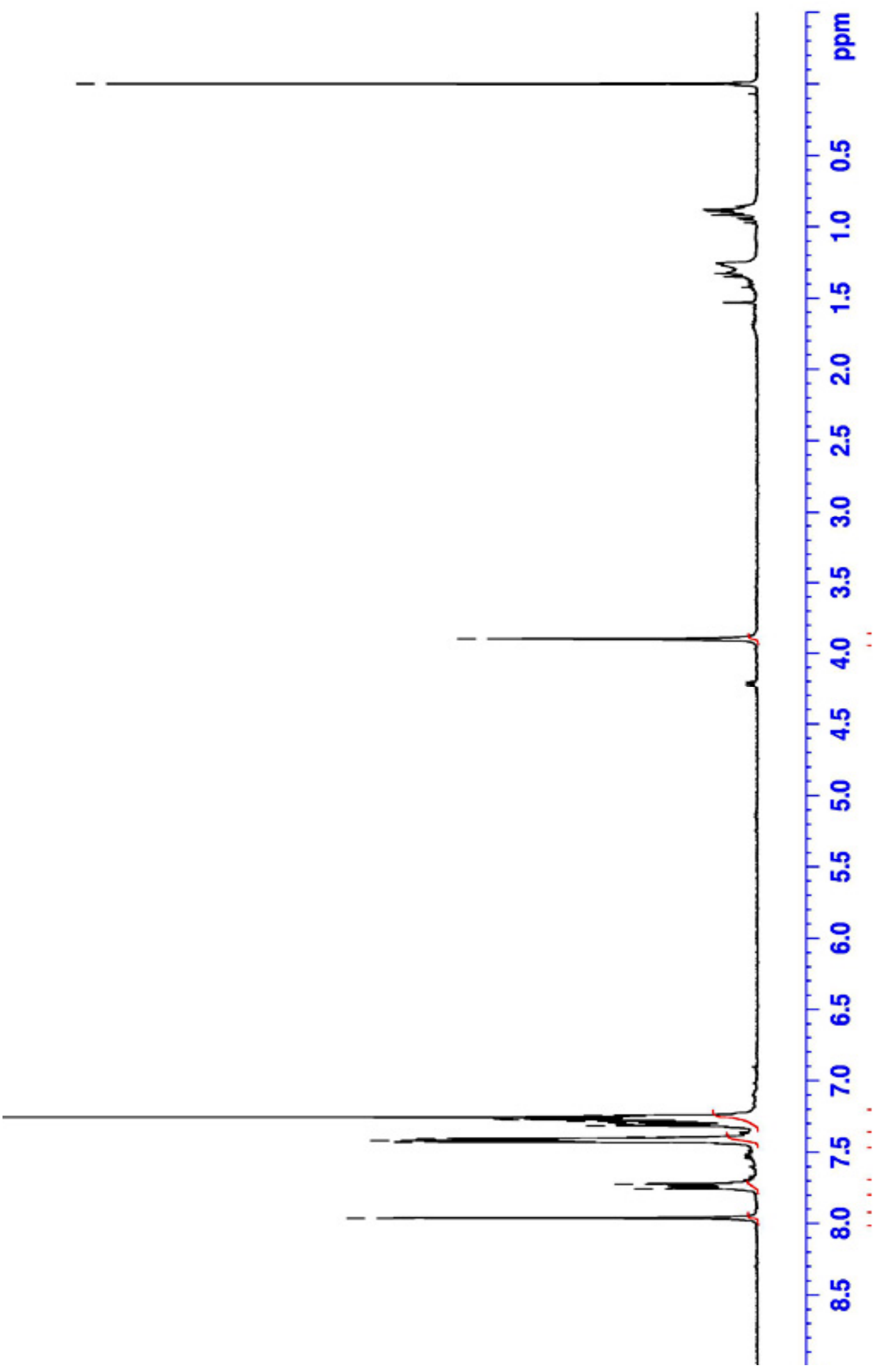

Figure S6. ${ }^{1} \mathrm{H}$ NMR spectra of $\mathbf{1}$ 


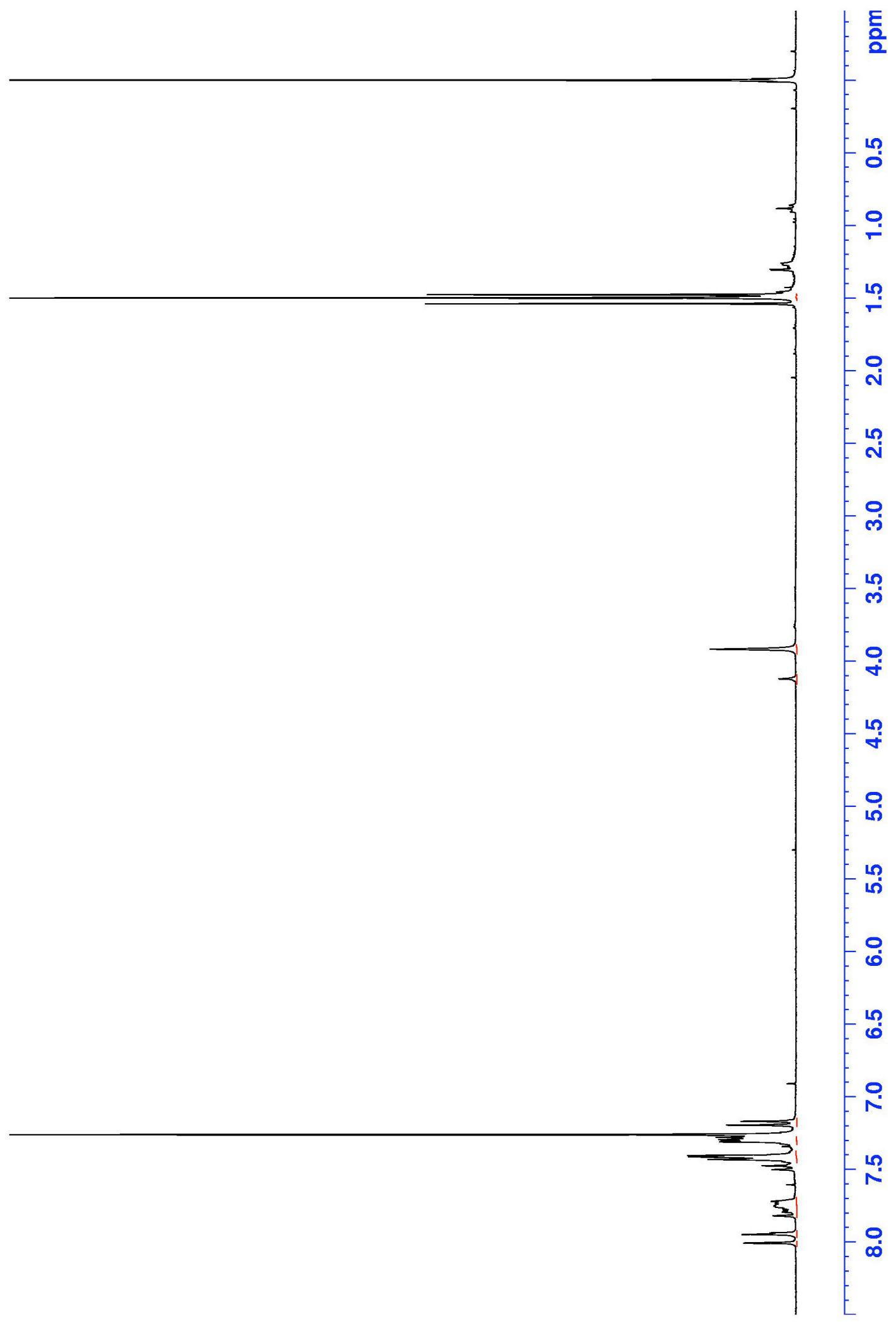

Figure S7. ${ }^{1} \mathrm{H}$ NMR spectra of 2 


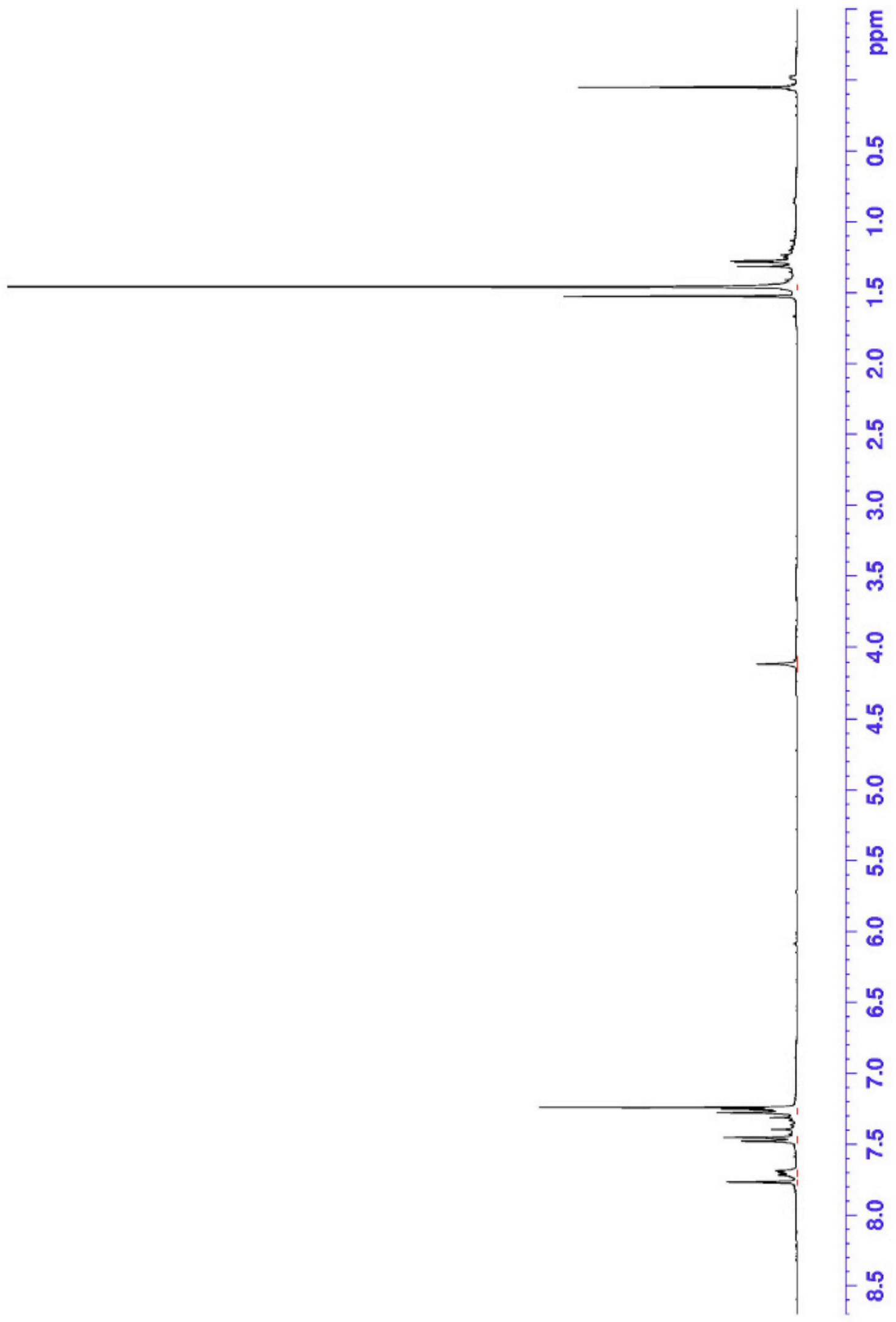

Figure S8. ${ }^{1} \mathrm{H}$ NMR spectra of $\mathbf{3}$ 